\title{
The potential of INTEGRAL for the detection of high redshift GRBs
}

\author{
J. Gorosabel ${ }^{1,2,3}$, N. Lund ${ }^{2}$, S. Brandt ${ }^{2}$, N. J. Westergaard ${ }^{2}$, and J. M. Castro Cerón ${ }^{3}$ \\ ${ }^{1}$ Instituto de Astrofísica de Andalucía (CSIC), Camino Bajo de Huétor, 24, 18008 Granada, Spain \\ e-mail: jgu@iaa.es \\ 2 Danish Space Research Institute, Juliane Maries Vej, 30, 2100 København Ø, Denmark \\ 3 Space Telescope Science Institute, 3.700 San Martin Drive, Baltimore, MD 21218-2463, USA
}

Received 10 October 2003 / Accepted 12 July 2004

\begin{abstract}
We discuss INTEGRAL's ability to detect a high redshift population of Gamma-Ray Bursts (GRBs) in comparison to other high-energy missions. Emphasis is placed on the study of the relative capabilities of IBIS on board INTEGRAL with respect to SWIFT and HETE 2 in detecting a high redshift population of GRBs. We conclude that, if the GRB rate is proportional to the star formation rate, INTEGRAL's ability to study GRBs are complementary to the ones of missions like SWIFT and HETE 2, devoted to prompt localisations of GRBs. Whereas SWIFT and HETE 2 would detect a higher number of GRBs than INTEGRAL, IBIS might be able to detect high redshift $(z \gtrsim 7)$ GRBs, unreachable by SWIFT and HETE 2 . We discuss the relevance of performing near-infrared (NIR) observations of the INTEGRAL GRBs and the strategy that large-class telescopes might follow.
\end{abstract}

Key words. gamma rays: bursts

\section{Introduction}

Gamma-Ray Bursts (GRBs) appear as brief $\left(10^{-3} \mathrm{~s}<\right.$ duration $<1000 \mathrm{~s}$ ) flashes of cosmic high-energy photons, emitting the bulk of their energy above $\approx 0.1 \mathrm{MeV}$. Among the thousands of GRBs detected in $\gamma$-rays since 1967 (Klebesadel et al. 1973) only $\sim 50$ GRBs have been identified at optical wavelengths ${ }^{1}$. For 36 of them spectroscopic redshifts have been measured, ranging from $z=0.0085$ (Galama et al. 1998) to $z=4.50$ (Andersen et al. 2000).

A canonical model has emerged for powering long GRBs (durations $\gtrsim 2$ s): collapse of a massive star onto a black hole. This leads to an intense flash of $\gamma$-ray photons followed by an expanding fireball which emits radiation at lower frequencies (Woosley 1993). The spectroscopic association of GRB 030329 with the type $\mathrm{I}_{c}$ SN $2003 \mathrm{dh}$ strongly supports this framework (Hjorth et al. 2003; Stanek et al. 2003). Short GRBs (durations $\lesssim 2 \mathrm{~s}$ ) have not yet shown a conclusive optical counterpart (see Castro-Tirado et al. 2002 for a further discussion).

We know today that most long duration GRBs originate at $z>0.5$. In fact, it is thought that their intrinsic brightness would allow us to detect these events at epochs corresponding to the formation of the earliest stellar populations. Thus, they may be used as probes of the first stages of star formation and their spectra may reveal the early heavy-element enrichment of the interstellar medium (ISM). GRBs caused by the exploding

\footnotetext{
${ }^{1}$ See http://www.mpe.mpg.de/ jcg/grbgen.html
}

primitive population of stars are suggested to be detectable up to redshifts $z \sim 30$ (Lamb \& Reichart 2000).

ESA's INTEGRAL satellite offers unique capabilities for the detection of GRBs thanks to its high sensitivity and imaging capabilities at $\gamma$-ray, X-ray and optical frequencies. INTEGRAL is the first $\gamma$-ray spacecraft that combines imaging instruments of high precision and a continuous real time telemetry link. Developments in the GRB field over the past few years have made it increasingly clear that INTEGRAL, and especially the IBIS $^{2} \gamma$-ray imager, could make a significant contribution. IBIS is the coded mask telescope on board INTEGRAL dedicated to imaging over a wide (15 keV-10 MeV) energy range (Ubertini et al. 2003). To date, neither of the three classical INTEGRAL GRBs (considering GRB 031203 as an X-ray flash) with optical counterparts identified have had their redshift measured (Götz et al. 2003; Castro-Tirado et al. 2003; Masetti et al. 2004).

In Sect. 2 we perform a rough comparison of INTEGRAL's sensitivity with respect to other $\gamma$-ray and X-ray missions/instruments. Section 3 studies in more detail the ability of the most sensitive high-energy missions/instruments (INTEGRAL/IBIS, HETE 2/WXM and SWIFT/BAT) to detect high redshift GRBs. Further, in Sect. 4 we focus on the INTEGRAL/IBIS and SWIFT/BAT relative number of high redshift GRB detections. Section 5 emphasises the relevance

\footnotetext{
${ }^{2}$ Imager on board the INTEGRAL satellite.
} 
Table 1. Theoretical estimations of several mission/instrument capabilities.

\begin{tabular}{lccccc}
\hline \hline Mission/Instrument & $\begin{array}{c}\text { Area } \\
\mathrm{cm}^{2}\end{array}$ & $\begin{array}{c}\text { Coverage } \\
\% \text { of } 4 \pi \text { str. }\end{array}$ & $\begin{array}{c}\text { Energy band } \\
(\mathrm{keV})\end{array}$ & $\begin{array}{c}\text { Orbit } \\
\text { efficiency }\end{array}$ & $\begin{array}{c}\text { Relative } \\
\text { sensitivity }\end{array}$ \\
\hline SWIFT/BAT & 5200 & $14^{\star}$ & 15 to 150 & 0.6 & 1.0 \\
INTEGRAL/IBIS (ISGRI) & 3000 & $0.9^{\star}$ & 15 to 150 & 0.8 & 3.0 \\
\hline HETE 2/WXM & 360 & 13 & 2 to 25 & 0.5 & 1.0 \\
INTEGRAL/JEM-X & 1000 & 0.1 & 2 to 35 & 0.8 & 19.0 \\
SAX/WFC & $530(\times 2)$ & $2(\times 2)$ & 2 to 30 & 0.5 & 3.1 \\
\hline
\end{tabular}

$\star$ Half coded field of view (FOV).

$\dagger$ Estimate based on the $P_{\text {ins }} \sim \sqrt{\Omega / A}$ recipe.

of the near-infrared (NIR) observations to detect high redshift afterglows. Section 6 presents the conclusions of our study.

\section{Sensitivity comparisons: INTEGRAL expectations}

In Table 1 we provide the main performances of a number of different space missions/instruments with capabilities for GRB research. The missions/instruments are divided in two groups depending on their energy range. In the first group the INTEGRAL/IBIS sensitivity is normalized with respect to the Burst Alert Telescope (BAT) $\gamma$-ray instrument on board $\mathrm{SWIFT}^{3}$. In the second group the INTEGRAL/JEM-X ${ }^{4}$ and $\mathrm{SAX} / \mathrm{WFC}^{5}$ rough sensitivities are given compared to the HETE 2/WXM ${ }^{6}$ one. Because we concentrate on determining the relative number of detections for several missions/instruments (see Sect. 4) we will not perform a dedicated calculation of the individual instrumental sensitivities; that goes beyond the scope of our study (see Band 2003 for a detailed analysis).

In order to compare the sensitivities of different missions, Band (2003) emphasised the need to express them in terms of a common energy band. Band (2003) determined the peak photon flux threshold (hereafter named $P_{\text {ins }}$, measured in ph $\mathrm{cm}^{-2} \mathrm{~s}^{-1}$ ), for several detectors, including HETE 2/WXM, SAX/WFC and SWIFT/BAT. INTEGRAL/IBIS and INTEGRAL/JEM-X were not considered in that study. In Band (2003) all the detection thresholds are normalised to the 1-1000 keV energy band, making several assumptions about the detectors' properties (accumulation time, detector response matrix, background model) and the GRBs' spectra ${ }^{7}$.

\footnotetext{
${ }^{3}$ Launch scheduled for the second half of 2004. Hereafter, when we refer to SWIFT we will focus only on BAT (which provides the GRB triggers for the mission) and not on the X-ray and $U V /$ Optical Telescopes on board SWIFT. See Gehrels et al. (2004) for further details.

${ }^{4}$ Joint European Monitor for X-rays, on board INTEGRAL.

5 Wide Field Cameras on board BeppoSAX.

6 The Wide X-ray Monitor is the most sensitive instrument on board HETE 2. The rest of the HETE 2 instrumentation will not be considered.

7 In Band (2003) the GRB spectra are described by the Band function (Band et al. 1993), where the photon number flux is given by:

$$
N(E)= \begin{cases}N_{0}\left(\frac{E}{100 \mathrm{keV}}\right)^{-\alpha_{1}} \exp \left(-\frac{E}{E_{0}}\right) & E \leq E_{b} \\ N_{0}\left(\frac{E_{b}}{100 \mathrm{keV}}\right)^{\alpha_{2}-\alpha_{1}}\left(\frac{E}{100 \mathrm{keV}}\right)^{-\alpha_{2}} \exp \left(\alpha_{1}-\alpha_{2}\right) & E>E_{b},\end{cases}
$$
}

Based on Figs. 5 and 7 in Band (2003) we assumed detection thresholds of $P_{\text {HETE } 2 / \mathrm{WXM}}^{(1-1000) \mathrm{kV}}=4 \mathrm{ph} \mathrm{cm}^{-2} \mathrm{~s}^{-1}$ for

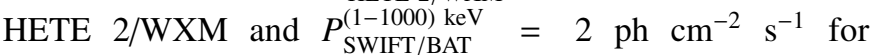
SWIFT/BAT. These two thresholds are valid for a broad range of $E_{p}$ values of around several hundreds of $\mathrm{keV}$ (a typical range for GRBs) and refer to the $1-1000 \mathrm{keV}$ energy band. We note that $E_{p}$ is the spectral maximum in the $v F_{v}$ (or equivalent $N(E) E^{2}$ ) representation. Many studies have reported results in the $50-300 \mathrm{keV}$ band, mainly because this band corresponds to the sensitivity range of $\mathrm{BATSE}^{8}$. Thus, in the present work we decided to carry out the sensitivity comparisons in a common 50-300 keV reference energy band. The selection of the energy band is arbitrary and does not affect our results, since it only introduces a constant multiplicative factor on the $P_{\text {ins }}$ values calculated for each mission/instrument (as well as on the luminosity, $L$, as it will be shown in Eq. (2)).

Setting $\alpha_{1}=\alpha_{2}=1.5$ (see Sect. 3), we calculated a flux ratio $P^{(50-300) \mathrm{keV}} / P^{(1-1000) \mathrm{keV}}=0.09$. For a power law spectrum this detection threshold ratio is independent of $E_{p}$ (which would correspond to a constant straight line in Fig. 1 of Band 2003). By transforming the assumed 1-1000 keV thresholds, the derived 50-300 keV sensitivities for HETE 2/WXM and SWIFT/BAT become $P_{\text {HETE } 2 / \mathrm{WXM}}^{(50-30) \mathrm{kV}}=0.35 \mathrm{ph} \mathrm{cm}^{-2} \mathrm{~s}^{-1}$ and $P_{\mathrm{SWIFT} / \mathrm{BAT}}^{(50-300) \mathrm{keV}}=0.17 \mathrm{ph} \mathrm{cm}^{-2} \mathrm{~s}^{-1}$, respectively. We note that these sensitivity values refer to the peak flux.

Estimates carried out for the IBAS $^{9}$ sensitivity yield a threshold of $\sim 0.14-0.22 \mathrm{ph} \mathrm{cm}^{-2} \mathrm{~s}^{-1}$ for INTEGRAL/IBIS (the ISGRI part) in the 20-200 keV energy band (Mereghetti et al. 2003). Hereafter we assume a conservative INTEGRAL/IBIS sensitivity limit of $P_{\text {INTEGRAL/IBIS }}^{(20-200) \mathrm{kV}}=0.22 \mathrm{ph} \mathrm{cm}^{-2} \mathrm{~s}^{-1}$, which corresponds to $P_{\text {INTEGRAL/IBIS }}^{(50-300) \mathrm{kV}}=0.12 \mathrm{ph} \mathrm{cm}^{-2} \mathrm{~s}^{-1}$, assuming $\alpha_{1}=\alpha_{2}=1.5$. Therefore, the INTEGRAL/IBIS capabilities of detecting high redshift GRBs relative to SWIFT/BAT (presented in Sect. 4) have to be considered as a lower estimate of the actual INTEGRAL/IBIS potential.

An alternative, simplified way to verify the photon peak flux thresholds for the instruments not considered by Band (2003) (like INTEGRAL/IBIS and INTEGRAL/JEM-X)

where $E_{b}=\left(\alpha_{2}-\alpha_{1}\right) E_{0}$, and $E_{0}$ determines the maximum of $N(E) E^{2}$ given by $E_{p}=\left(2-\alpha_{1}\right) E_{0} . \alpha_{1}$ and $\alpha_{2}$ are the photon spectral indexes at frequencies below and above $E_{b}$.

8 The Burst And Transient Source Experiment; it operated on board the Compton Gamma-Ray Observatory between 1991 and 2000.

${ }^{9}$ INTEGRAL Burst Alert System. 
would be to assume that the sensitivity threshold $P_{\text {ins }}$ for a GRB is proportional to the square root of the background count rate and inversely proportional to the square root of the detector area. Thus we have $P_{\text {ins }} \propto \sqrt{\Omega / A}$, where $\Omega$ is the sky coverage and $A$ is the detector area. This assumption would yield a sensitivity ratio of 3.0 between INTEGRAL/IBIS and SWIFT/BAT (see Table 1) and, therefore, a threshold of $P_{\text {INTEGRAL/IBIS }}^{(50-300) \mathrm{keV}}=$ $0.06 \mathrm{ph} \mathrm{cm}^{-2} \mathrm{~s}^{-1}$.

In principle, the most reliable comparison that can be done in Table 1 is between SWIFT/BAT and INTEGRAL/IBIS (the ISGRI part), because they are based on very similar detector technologies (CdZnTe in SWIFT/BAT, CdTe in INTEGRAL/IBIS) and share a similar energy band. This simple estimate is a factor of two lower than the $P_{\text {INTEGRAL/IBIS }}^{(50-300) \mathrm{kV}}=$ $0.12 \mathrm{ph} \mathrm{cm}^{-2} \mathrm{~s}^{-1}$ threshold assumed for INTEGRAL/IBIS. Hence, it supports the use of this threshold as a conservative upper limit of the real INTEGRAL/IBIS sensitivity.

From the above estimates it is evident that INTEGRAL/IBIS will be the most sensitive GRB detector (at least in the 15-150 keV energy band) ever flown and not likely to be matched, sensitivity-wise, by any other mission within the coming decade.

Rescaling the HETE 2/WXM threshold with the $P_{\text {ins }} \propto$ $\sqrt{\Omega / A}$ recipe we obtain a sensitivity of $P_{\text {INTEGRAL } / \text { JEM-X }}^{(50-300) \mathrm{kVV}}=$ $0.02 \mathrm{ph} \mathrm{cm}^{-2} \mathrm{~s}^{-1}$. However, the difference in detector technologies of INTEGRAL/JEM-X with respect to HETE 2/WXM makes this number an uncertain estimate of the actual INTEGRAL/JEM-X sensitivity. Furthermore, the on-flight INTEGRAL/JEM-X performances have been changed during the first months of the INTEGRAL mission, so its real sensitivity is well above $0.02 \mathrm{ph} \mathrm{cm}^{-2} \mathrm{~s}^{-1}$. The reduced number of bursts that INTEGRAL/JEM-X will detect (a very few per year) does not support performing a specific calculation aimed at studying its capabilities for high redshift bursts.

On the other hand, an estimate based on Figs. 3 and 5 of Band (2003) yields a relative sensitivity of $\sim 3-4$ between HETE 2/WXM and SAX/WFC. This is in agreement with the HETE 2/WXM vs. SAX/WFC relative sensitivity estimate given by the $P_{\text {ins }} \propto \sqrt{\Omega / A}$ expression (see Table 1 ).

\section{Detectability of a high redshift population of GRBs}

We have selected the most sensitive missions/instruments (present: INTEGRAL/IBIS, HETE 2/WXM, and future: SWIFT/BAT) to calculate their ability to detect a high redshift population of bursts. To estimate the number of GRBs that these missions/instruments will detect we assume that:

- GRB spectra can be described by power laws $\left(F_{v} \sim v^{-\alpha}\right)$. After exploring the impact of $\alpha$ on the final results (which do not change qualitatively) and for simplicity, we have assumed a value of $\alpha=1.5$, typical of GRBs (van Paradijs et al. 2000).

- The GRB rate is proportional to the star formation rate (SFR) in the Universe. The SFR considered is the one given by Rowan-Robinson $(1999,2001)$ for $z<5$ and the one calculated by Gnedin \& Ostriker (1997) for $z \geq 5$ (see Fig. 1).

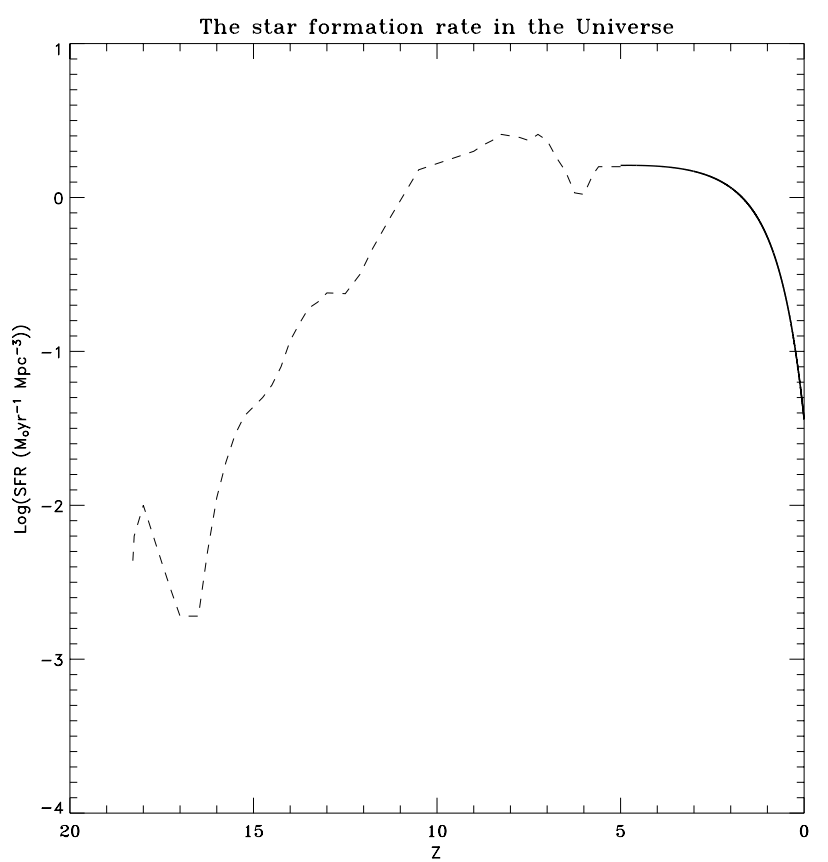

Fig. 1. The SFR in the Universe as a function of the redshift. The dashed line represents the SFR derived from numerical simulations for $z \geq 5$ (Gnedin \& Ostriker 1997). The solid line shows the SFR at the $z<5$ region based on observational estimates (Rowan-Robinson 1999, 2001). The transition between the two regions has been smoothed.

- The GRB peak (isotropic) photon luminosity function is given by:

$S(L)= \begin{cases}L^{\beta} & L_{\min }<L<L_{\max } \\ 0 & \text { Otherwise }\end{cases}$

$L$ being the peak photon luminosity and $\beta$ the luminosity function index. $L_{\min }, L_{\max }$ determine the width of the luminosity function. We have assumed a value of $\beta=-1$.

- Although the effect of several Universe models has been checked, we choose to use the most popular cosmological parameters in this paper: $\Omega_{\mathrm{m}}=0.3, \Omega_{\Lambda}=0.7, H_{0}=$ $65 \mathrm{~km} \mathrm{~s}^{-1} \mathrm{Mpc}^{-1}$.

For the above assumptions the differential GRB detection rate at a given peak photon flux $P$ in a detector $\left(\mathrm{ph} \mathrm{cm}^{-2} \mathrm{~s}^{-1}\right)$ is given by the following convolution integral:

$N_{\mathrm{GRB}}(P)=C \Omega \epsilon \int_{0}^{\infty} R_{\mathrm{GRB}} S(L) \mathrm{d} L$,

where $\epsilon$ is the efficiency of the orbit, $\Omega$ is the instrumental coverage of the sky and $R_{\mathrm{GRB}}$ is the GRB detection rate if they were standard candles, i.e., $R_{\mathrm{GRB}}=\frac{\operatorname{SFR}(z)}{(1+z)} \frac{\mathrm{d} V(z)}{\mathrm{d} z} \frac{\mathrm{d} z}{\mathrm{~d} P}$, being $V$ the comoving volume. The value of the proportionality constant $C$ is unknown. Figure 2 displays $N_{\mathrm{GRB}}(P)$ as well as the detection thresholds of several high-energy missions/instruments.

The relationship between $L, z$ and $P$ is given by the following expression:

$P=\frac{L}{4 \pi D(z)^{2}(1+z)^{\alpha}}$ 


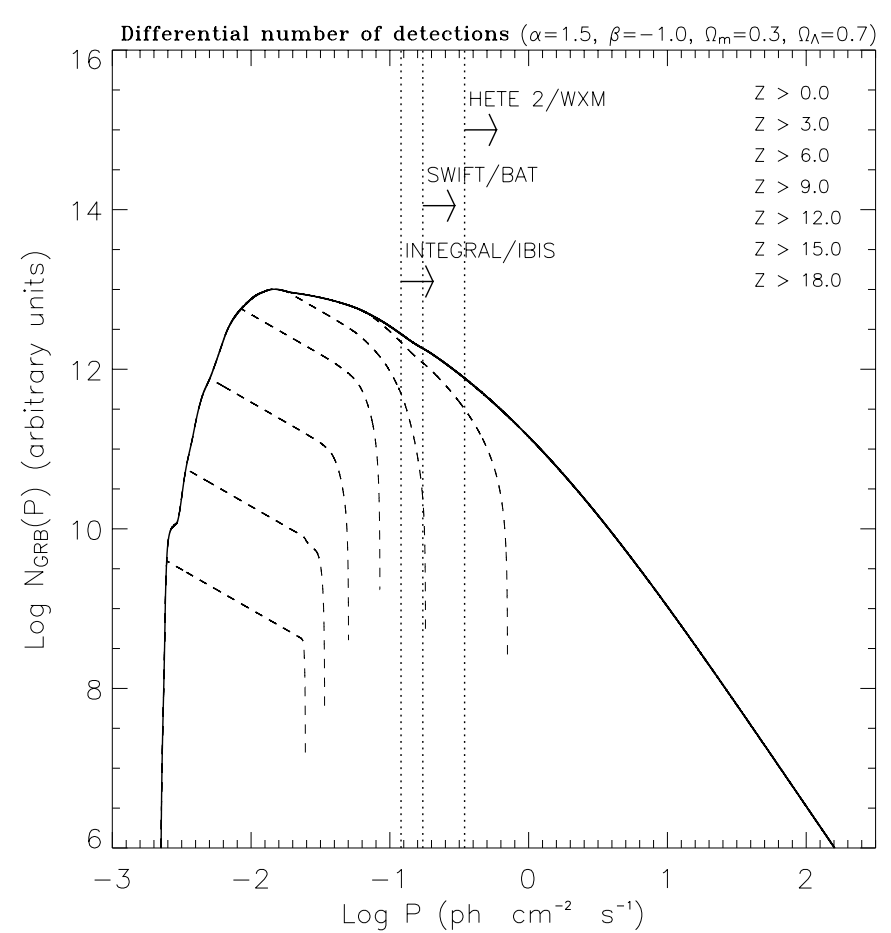

Fig. 2. Differential peak photon flux distribution of GRBs. The solid curve shows the differential peak photon flux distribution if all redshifts are considered, i.e., $N_{\mathrm{GRB}}(P)$. The dashed curves represent the differential peak photon flux distribution of GRBs when only GRBs with $z>z_{\text {edge }}$ are taken into account, i.e., $N_{\mathrm{GRB}}\left(P, z_{\text {edge }}\right)$. The intention of this figure is to show how the Gaussian-like solid curve displaces leftwards (low $P$ values) as $z_{\text {edge }}$ increases. The dashed lines plotted in the figure are shown as an example, using arbitrary values of $z_{\text {edge, }}$, not marked for simplicity. As $z_{\text {edge }}$ increases the closer GRBs (and therefore larger $P$ bursts) are left out (right tail of the Gaussian-like distribution). The vertical lines represent the detection thresholds for the different missions/instruments, showing the arrows the detectability region.

where $D(z)$ is the comoving distance. In our calculations different values of $\alpha, L_{\min }, L_{\max }, \beta, \Omega_{\Lambda}$ and $\Omega_{\mathrm{m}}$ are considered. Variations of $\alpha, \beta, \Omega_{\Lambda}$ and $\Omega_{\mathrm{m}}$ do not change the final result qualitatively. On the other hand, the variations of $L_{\min }, L_{\max }$ are more relevant for determining the number of high redshift GRB detections. We consider the pessimistic case of a relatively faint and narrow luminosity function $S(L)$ defined by $L_{\min }=10^{57.5} \mathrm{ph} \mathrm{s}^{-1}$ and $L_{\max }=10^{58.5} \mathrm{ph} \mathrm{s}^{-1}$. The $L_{\min }$ and $L_{\max }$ values used in our study correspond to the narrowest $S(L)$ among the ones used by Lamb \& Reichart (2000), and consistent with the observed peak photon luminosity distribution (the measured $S(L)$ is at least 1.7 times wider; Stern et al. 2002) ${ }^{10}$. The assumption of a wider $S(L)$ would broaden the Gaussianlike curves (both solid and dashed) displayed in Fig. 2, extending their tails to higher $P$ values and therefore enhancing the number of GRBs detected at very high redshift.

\footnotetext{
${ }^{10}$ Our $L_{\min }$ and $L_{\max }$ values are also in agreement with the empirical peak photon luminosity function used as reference by Lamb \& Reichart (2000); $\log L=58.1 \pm 0.7$.
}

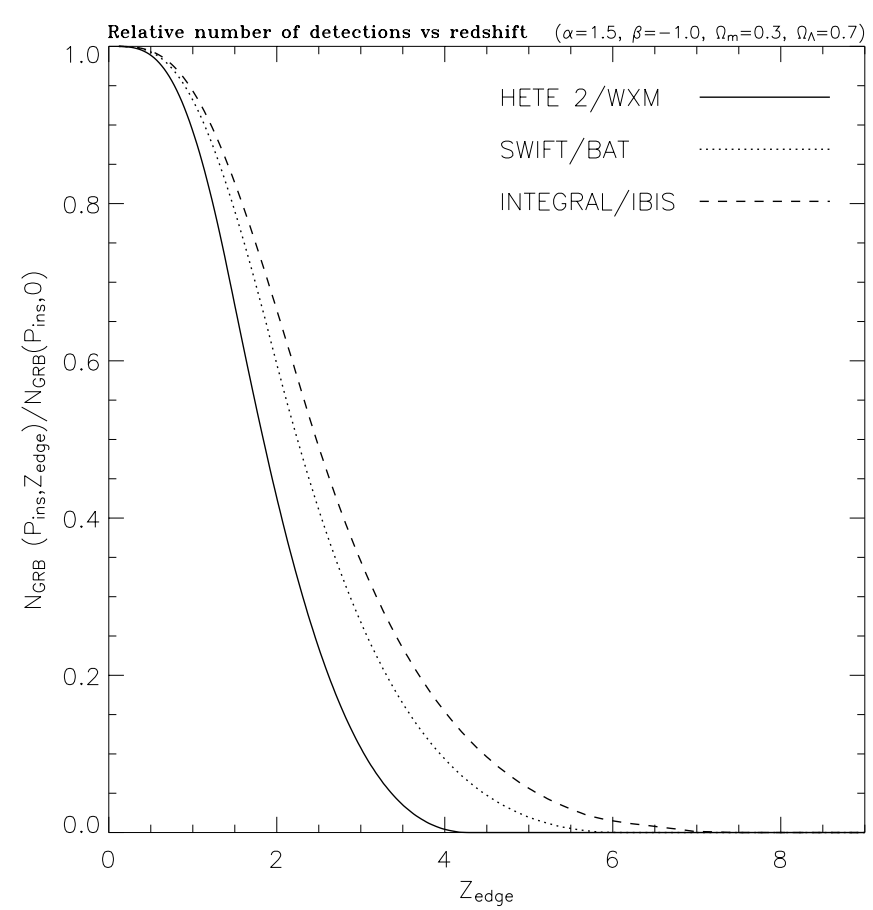

Fig. 3. Relative number of detections as a function of the redshift. This plot shows, for several high-energy missions/instruments, the fraction of the detected GRBs that have a redshift larger than $z_{\text {edge }}$.

We can calculate the contribution to (1) by GRBs with redshift larger than $z_{\text {edge }}$ (see dashed curves of Fig. 2), using:

$N_{\mathrm{GRB}}\left(P, z_{\text {edge }}\right)=C \Omega \epsilon \int_{0}^{\infty} H\left(z(L), z_{\text {edge }}\right) R_{\mathrm{GRB}} S(L) \mathrm{d} L$,

where $H\left(z(L), z_{\text {edge }}\right)$ is a step function that vanishes unless $z(L)>z_{\text {edge }}$. Obviously, $N_{\mathrm{GRB}}(P)=N_{\mathrm{GRB}}(P, 0)$, and $\frac{N_{\mathrm{GRB}}\left(P, z_{\text {edge }}\right)}{N_{\mathrm{GRB}}(P)} \leq 1$. Last, we can calculate the number of GRBs detected above a given instrumental peak photon flux threshold $P_{\text {ins }}$ that have redshifts larger than $z_{\text {edge }}$ :

$N_{\mathrm{GRB}}\left(P_{\text {ins }}, z_{\text {edge }}\right)=\int_{P_{\text {ins }}}^{\infty} N_{\mathrm{GRB}}\left(P, z_{\text {edge }}\right) \mathrm{d} P$.

Not knowing the proportionality constant $C$ of (1), we cannot derive an absolute value for $N_{\mathrm{GRB}}\left(P_{\text {ins }}, z_{\text {edge }}\right)$. However, we can determine the relative quantity $\frac{N_{\mathrm{GRB}}\left(P_{\text {ins }}, z_{\text {edge }}\right)}{N_{\mathrm{GRB}}\left(P_{\text {ins }}, 0\right)}$, which provides the proportion of detections that have a redshift larger than $z_{\text {edge }}$ (see Fig. 3).

\section{INTEGRAL/IBIS vs. SWIFT/BAT; comparison of the number of GRB detections}

Figure 3 shows the relative number of detections (given by $\left.\frac{N_{\mathrm{GRB}}\left(P_{\text {ins }}, z_{\text {edge }}\right)}{N_{\mathrm{GRB}}\left(P_{\text {ins }}, 0\right)}\right)$, as a function of the redshift, for HETE $2 / \mathrm{WXM}$, SWIFT/BAT and INTEGRAL/IBIS. As it is shown, $\sim 15 \%$ of the GRBs detected by INTEGRAL/IBIS will have a redshift larger than 4. For SWIFT/BAT the $z>4$ population will be $\sim 10 \%$ of the total number of detections. In the case of INTEGRAL/IBIS the tail of $\frac{N_{\mathrm{GRB}}\left(P_{\text {ins }}, z_{\text {edge }}\right)}{N_{\mathrm{GRB}}\left(P_{\text {ins }}, 0\right)}$ extends even up to redshifts of $z_{\text {edge }}>7$. SWIFT/BAT and HETE 2/WXM will detect a closer population of bursts, specially HETE 2/WXM. 
Relative number of detections between INTEGRAL/IBIS and SWIFT/BAT

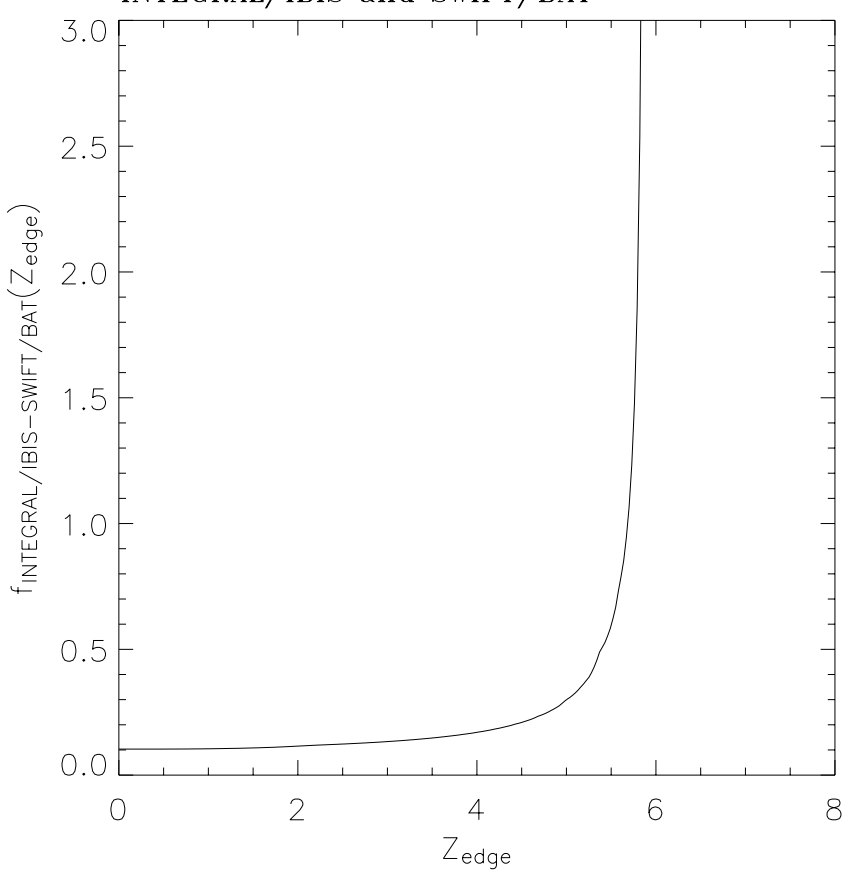

Fig. 4. The GRB detection ratio of INTEGRAL/IBIS with respect to SWIFT/BAT as a function of the GRB population redshift.

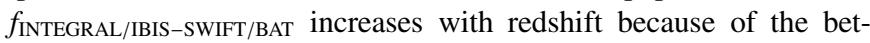
ter sensitivity for high redshift GRBs of INTEGRAL/IBIS. At redshifts $z \lesssim 5.6$ the larger field of view of SWIFT dominates, making $f_{\text {INTEGRAL/IBIS-SWIFT/BAT }}<1$.

HETE 2/WXM is the least sensitive GRB mission, being constrained to detect bursts with redshifts $z<4.3$. This prediction is in agreement with the maximum redshift measured for a HETE 2/WXM GRB ${ }^{11}$. Therefore we will not consider HETE 2/WXM in the following study, aimed at calculating the relative number of GRB detections as a function of the redshift. We will concentrate on comparing INTEGRAL/IBIS and SWIFT/BAT capabilities. As we previously noted, the similar energy range and detector technologies of INTEGRAL/IBIS and SWIFT/BAT suggest a reliable comparison.

To determine the relative number of detections between two missions, $\mathrm{A}$ and $\mathrm{B}$, the following calculation has to be performed:

$$
f_{\mathrm{A}-\mathrm{B}}\left(z_{\text {edge }}\right)=\frac{\Omega_{\mathrm{A}} \epsilon_{\mathrm{A}} \int_{P_{\mathrm{A}}}^{\infty} \int_{0}^{\infty} H\left(z(L), z_{\text {edge }}\right) R_{\mathrm{GRB}} S(L) \mathrm{d} L \mathrm{~d} P}{\Omega_{\mathrm{B}} \epsilon_{\mathrm{B}} \int_{P_{\mathrm{B}}}^{\infty} \int_{0}^{\infty} H\left(z(L), z_{\text {edge }}\right) R_{\mathrm{GRB}} S(L) \mathrm{d} L \mathrm{~d} P} .
$$

This function will give the relative number of GRB detections with $z>z_{\text {edge }}$. We have applied (2) to derive the detection ratio between INTEGRAL/IBIS and SWIFT/BAT as a function of the GRB population redshift $\left(f_{\text {INTEGRAL/IBIS-SWIFT/BAT }}\left(z_{\text {edge }}\right)\right)$.

As it is shown in Fig. 4, for zedge $<5.6$ $f_{\text {INTEGRAL/IBIS-SWIFT/BAT }}<1$. In other words, at low redshifts the large field of view (FOV) of SWIFT/BAT, in comparison to INTEGRAL/IBIS, dominates the number of detections. On the

\footnotetext{
11 Among the 12 HETE 2/WXM GRBs with confirmed spectroscopic redshifts to date, the maximum redshift is reached for GRB 030323 at $z=3.372$ (Vreeswijk et al. 2004).
}

other hand, for $z_{\text {edge }}>5.6$, INTEGRAL/IBIS sensitivity becomes the dominating factor and $f_{\text {INTEGRAL/IBIS-SWIFT/BAT }}>1$. Thus, from the point of view of the ground based strategy of detecting $z \gtrsim 6$ afterglows, it might be more efficient to observe INTEGRAL/IBIS GRBs than to observe SWIFT/BAT bursts. If we are interested in using GRBs to study the reionisation epoch that occurred at $z \sim 7$ (Loeb \& Barkana 2001), then it would be relevant to prioritise the follow up of INTEGRAL/IBIS GRBs. Nonetheless we emphasise that INTEGRAL/IBIS high redshift detectability predictions are based on low number statistics, so they are subjected to large fluctuations.

The self consistency of our procedure can be checked by studying the prediction of expression (3) for the particular case when $z_{\text {edge }}=0$. If we consider $z_{\text {edge }}=0$, then $f_{\text {INTEGRAL/IBIS-SWIFT/BAT }}(0)$ gives us the fraction of GRBs detected with $z>0$, i.e., considering all the detections independently of their redshifts. We predict a value of $f_{\text {INTEGRAL/IBIS-SWIFT/BAT }}(0)=1 / 9.7$ for the fraction of the total number of GRBs detected by INTEGRAL/IBIS compared to SWIFT/BAT (see Fig. 4 at $z_{\text {edge }}=0$ ). The number of GRBs currently detected by INTEGRAL/IBIS is $\sim 1$ GRB per month (Mereghetti et al. 2003), whereas the last updated estimates for the SWIFT/BAT detection rate yield $\sim 110$ GRBs per year (Heyl 2003). This gives a fraction of $\sim 1 / 9.2$ for the number of detected GRBs, in agreement with our prediction.

Although INTEGRAL/JEM-X FOV and sensitivity are less suitable than the ones of INTEGRAL/IBIS to detect GRBs, the spectral peak of the high redshift GRBs (usually at $100-1200 \mathrm{keV}$ ) will be in the detection range of INTEGRAL/JEM-X. So given that INTEGRAL/JEM-X is coaligned with INTEGRAL/IBIS, it might be also useful to detect the redshifted prompt $\gamma$-ray component.

\section{NIR observations for INTEGRAL GRBs}

The most significant inconvenience in following up INTEGRAL GRB afterglows is the large fraction of the mission's time devoted to scan the extinguished Galactic centre/plane. An additional problem (not specific to INTEGRAL, like the previous one) for detecting high redshift GRBs comes from the Ly- $\alpha$ blanketing effect that strongly attenuates the radiation observed at $\lambda<1216 \times(1+z) \AA$. Both drawbacks can be mitigated if ground-based searches are carried out in the NIR, specially in the $K$-band, where $i$ ) the Galactic extinction drops by a factor $\sim 6$ compared to the optical, and ii) the Ly- $\alpha$ blanketing problem is eliminated up to very high redshifts $(z \sim 17)$.

There are further advantages to searching for high redshift afterglows in the NIR. Because of their fading behaviour (typically, GRB afterglows decay following a power law of the form $F_{v} \sim t^{-\delta}$, where $t$ is the time since the onset of the $\gamma$-ray event and $\delta$ the decay index) the time dilation effect increases the observed flux at a fixed time of observation after the GRB. Thus current and future ground-based NIR facilities could be able to detect high redshift afterglows that are invisible in the optical bands. 


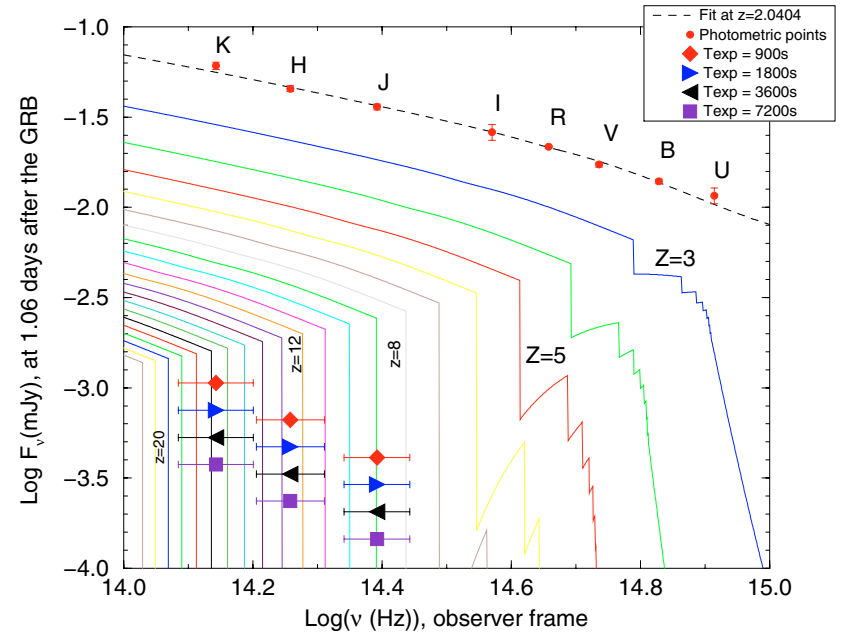

Fig. 5. The evolution of the observed flux of a typical GRB afterglow (GRB 000301c) when it is redshifted from $z=2.0404$ (spectroscopic redshift; Jensen et al. 2001) up to $z=22$. The circles show the photometric measurements of the afterglow from the $U$ to the $K$-band, taken 1.06 days after the GRB. The dashed line represents the SED fitted to the photometric points (see main text). The rhomboids, triangles and squares show the $J H K$-band sensitivities $(5 \sigma)$ foreseen for the future GTC $10 \mathrm{~m}$ telescope (+ EMIR instrument), for several exposure times. Afterglows can be detected in the three $J H K$ bands up to $z=9$, with only a exposure time of $900 \mathrm{~s}$ per filter. $K$-band counterparts would be detectable up to $z \sim 17$. For $T_{\exp }>900 \mathrm{~s}$ the detectability of the GRB 000301c afterglow with the GTC (+EMIR) is dominated by the Ly- $\alpha$ blanketing effect, and is basically independent of the exposure time employed in the observations. The Ly- $\alpha$ blanketing absorption has been modeled following Madau (1995).

As an example, in Fig. 5 we display the very well sampled spectral energy distribution (SED) of the GRB 000301c afterglow (Jensen et al. 2001) for different redshifts, once the Ly- $\alpha$ blanketing absorption has been modeled (Madau 1995). The photometric point flux densities $\left(F_{v}\right.$; see dashed line in Fig. 5) have been fitted following an expression of the form $F_{v} \sim v^{\eta} \times 10^{-0.4 A_{v}}$, where $\eta$ is the afterglow power law spectral index and $A_{v}$ is the absorption in the GRB host galaxy at a frequency of $v . A_{v}$ has been parameterised in terms of $A_{\mathrm{V}}$ following a typical SMC extinction law (Pei 1992). For illustration purposes we have overplotted, for different exposure times, the $J H K$-band $5 \sigma$ sensitivities foreseen for the Espectrógrafo Multiobjeto Infrarrojo (EMIR) (Balcells 1998), currently being build for the $10 \mathrm{~m}$ Gran Telescopio Canarias (GTC) (Rodríguez Espinosa et al. 1998) $^{12}$.

We would like to emphasise several points. First, this simple redshifting exercise does not require any assumption on the star formation rate, luminosity function, etc. Second, the GRB 000301c afterglow was not especially bright, so an intense afterglow (like GRB 990123; Castro-Tirado et al. 1999) could be detectable even at higher redshifts. Third, the photometric points displayed in the figure correspond to a fairly late epoch observation (they were acquired $\sim 1.06$ days after

\footnotetext{
12 For additional information, please, visit the following sites: http://www.ucm.es/info/emir/index_e.html, and also: http://www.gtc.iac.es/
}

the burst, time measured in the observer's frame), so afterglows observed a few hours (even minutes) after the burst would be reachable by smaller facilities (i.e., NIR robotic telescopes like BOOTES-IR or REM; Castro-Tirado et al. 2004, Zerbi et al. 2001, 2003).

As it is shown in Fig. 5, rapid NIR observations of afterglows performed with $10 \mathrm{~m}$ class telescopes (e.g., the GTC equipped with EMIR) would be dominated by the Ly- $\alpha$ blanketing effect since, above a given exposure time threshold $\left(T_{\exp } \sim 900 \mathrm{~s}\right.$ for the particular case of GRB 000301c and the GTC), the detectability is basically independent of the exposure time employed in the observations.

At high redshifts the observer would be sampling the UV part of the afterglow synchrotron spectrum where the extinction of the GRB environment is expected to be severe. Thus one of the major problems predicting the NIR detectability of a high redshift afterglow would come from the so far unknown extinction law and dust content describing both the local GRB environment and the large-scale line of sight properties within the host galaxy. Furthermore, the UV opacity in the environment close to the GRB progenitor might be timedependent. Hence, a detailed quantitative prediction of the number of afterglows detected in the NIR (beyond the scope of this work) would require modeling the physical processes describing the UV absorption of the afterglow radiation and its evolution at different scales (from several AU to $\mathrm{kpc}$ ) around the GRB progenitor.

\section{Discussion and conclusions}

In the present paper we study the capabilities of several missions/instruments to detect high redshift GRBs. At low and moderately high redshifts $(0<z \lesssim 5.6)$ the large FOV of SWIFT/BAT, compared to the instrumentation on board INTEGRAL, dominates the number of GRB detections. Nonetheless, the better sensitivity of IBIS makes INTEGRAL more efficient at detecting GRBs beyond $z \sim 5.6$.

In the most popular cosmological models, the first sources of light began at a redshift of $z=30$ and reionised most of the Universe by $z \sim 7$ (Loeb \& Barkana 2001). Thus the study of the Ly- $\alpha$ forest, present in the high redshift INTEGRAL/IBIS afterglow spectra, might constrain the epoch of such reionisation (Gunn \& Peterson 1965), probing the ionisation state of the intergalactic medium (IGM) as a function of redshift. According to our estimates this kind of study will be less productive if SWIFT/BAT (even more HETE 2/WXM), rather than INTEGRAL/IBIS, GRBs are followed up.

Further, optical/NIR spectroscopy of high redshift afterglows would provide additional information on the primitive IGM and ISM. High resolution spectroscopy of high redshift afterglows might reveal metal absorption lines, which could trace the metal enrichment history of the Universe. In principle this is the same technique as the one used for studying the damped Ly- $\alpha$ systems (DLAs), which are located in the line of sight of quasars. However GRBs are cleaner probes than quasars, because a brief phenomenon such as a GRB would not modify the state of the environment at large distances, as quasars do (the Strömgren sphere of high redshift quasars is 
of the order of Mpc; White et al. 2003). GRBs would allow to study the ISM of basically unaltered host galaxies, highly ionised if they had harboured a quasar during at least one million years. Moreover, GRBs are brighter (although for a short time) than quasars, so GRBs can probe the ISM of dustier galaxies.

Theoretical studies show that the birthrate of Population III stars produces a peak in the SFR in the Universe at redshifts $16 \lesssim z \lesssim 20$, while the birthrate of Population II stars produces a much larger and broader peak at redshifts $2 \lessgtr z \lessgtr 10$ (Valageas \& Silk 1999). If GRBs are produced by the collapse of massive stars, they are expected to occur at least at $z \sim 10$ and possibly up to $z \sim 15-20$. The detection of very high redshift GRBs would give us for the first time direct confirmation of the existence of the earliest stellar generations.

Rapid NIR observations are a complementary strategy to study high redshift INTEGRAL/IBIS GRBs. $K$-band observations performed with current and planned ground based NIR facilities might detect GRBs up to the theoretical limit imposed by the Ly- $\alpha$ blanketing $(z \sim 17)$. Realistically however the $z \sim 17$ upper limit ought to be decreased due to possible UV absorption present in the GRB host galaxy.

In conclusion, the INTEGRAL/IBIS's ability to study GRBs is complementary to the ones of instruments like SWIFT/BAT and HETE 2/WXM, devoted to prompt localizations of GRBs. Whereas SWIFT/BAT and HETE 2/WXM would detect more GRBs than INTEGRAL/IBIS, the latter might detect high redshift GRBs unreachable to the former. Future works might invert the logical outflow followed in the present paper. Once spectroscopic redshifts have been measured for a large sample of GRBs (and therefore the number of detected GRBs as a function of redshift is known), the equations could be inverted to obtain the SFR evolution with redshift. INTEGRAL, and especially IBIS, could be a very valuable tool to trace the SFR rate in the early Universe.

Acknowledgements. We thank our anonymous referee for fruitful and constructive comments. We are very grateful to David L. Band for helpful information used to determine the instrumental thresholds.

\section{References}

Andersen, M. I., Hjorth, J., Pedersen, H., et al. 2000, A\&A, 364, L54 Balcells, M. 1998, Ap\&SS, 263, 361
Band, D. L., Matteson, J., Ford, L., et al. 1993, ApJ, 413, 281

Band, D. L. 2003, ApJ, 588, 945

Castro-Tirado, A. J., Zapatero-Osorio, M. R., Caon, N., et al. 1999, Science, 283, 2069

Castro-Tirado, A. J., Castro Cerón, J. M., Gorosabel, J., et al. 2002, A\&A, 393, L55

Castro-Tirado, A. J., Gorosabel, J., Guziy, S., et al. 2003, A\&A, 411, L315

Castro-Tirado, A. J., et al. 2004, Proceedings of the I Reunión Nacional de Astrofísica Robótica, Mazagón (Spain), in press

Galama, T. J., Vreeswijk, P. M., van Paradijs, J., et al. 1998, Nature, 395,670

Gehrels, N., Chincarini, G., Giommi, P., et al. 2004, ApJ, in press, [arXiv: astro-ph/0405233]

Gnedin, N. Y., \& Ostriker, J. P. 1997, ApJ, 486, 581

Götz, D., Mereghetti, S., Hurley, K., et al. 2003, A\&A, 409, 831

Gunn, J. E., \& Peterson, B. A. 1965, ApJ, 142, 1633

Heyl, J. S. 2003, ApJ, 592, 401

Hjorth, J., Sollerman, J., Møller, P., et al. 2003, Nature, 423, 847

Jensen, B. L., Fynbo, J. P. U., Gorosabel, J., et al. 2001, A\&A, 370, 909

Klebesadel, R. W., Strong, I. B., \& Olson, R. A. 1973, ApJ, 182, L85

Lamb, D. Q., \& Reichart, D. E. 2000, ApJ, 536, L1

Loeb, A., \& Barkana, R. 2001, ARA\&A, 39, 19

Madau, P. 1995, ApJ, 441, 18

Masetti, N., Palazzi, E., Rol, E., et al. 2004, GCN Circ. \# 2515

Mereghetti, S., Götz, D., Borkowski, J., Walter, R., \& Pedersen, H. 2003, A\&A, 411, L291

Pei, Y. C. 1992, ApJ, 395, 130

Rodríguez Espinosa, J. M., Álvarez, P., \& Sánchez, F. 1998, Ap\&SS, 263,355

Rowan-Robinson, M. 1999, Ap\&SS, 266, 291

Rowan-Robinson, M. 2001, ApJ, 549, 745

Stanek, K. Z., Matheson, T., Garnavich, P. M., et al. 2003, ApJ, 591, L17

Stern, B. E., Tikhomirova, Ya., \& Svensson, R. 2002, ApJ, 573, 75

Ubertini, P., Lebrun, F., Di Cocco, G., et al. 2003, A\&A, 411, L131

Valageas, P., \& Silk, J. 1999, A\&A, 347, 1

van Paradijs, J., Kouveliotou, C., \& Wijers, R. A. M. J. 2000, ARA\&A, 38, 379

Vreeswijk, P. M., Ellison, S. L., Ledoux, C., et al. 2004, A\&A, 419, 927

White, R. L., Becker, R. H., Fan, X., \& Strauss, M. A. 2003, AJ, 126, 1

Woosley, S. E. 1993, ApJ, 405, 273

Zerbi, F. M., Chincarini, G., Ghisellini, G., et al. 2001, AN, 322, 275

Zerbi, F. M., Chincarini, G., Ghisellini, G., et al. 2003, SPIE, 4841, 737 